\title{
Vildagliptin and caloric restriction for cardioprotection in pre-diabetic rats
}

\author{
Pongpan Tanajak1,2,3, Hiranya Pintana1,2,3, Natthaphat Siri-Angkul1,2,3, \\ Juthamas Khamseekaew1,2,3, Nattayaporn Apaijai1,2,3, Siriporn C Chattipakorn1,3,4 \\ and Nipon Chattipakorn ${ }^{1,2,3}$
}

${ }^{1}$ Cardiac Electrophysiology Research and Training Center, Faculty of Medicine, Chiang Mai University, Chiang Mai, Thailand

2Cardiac Electrophysiology Unit, Department of Physiology, Faculty of Medicine, Chiang Mai University, Chiang Mai, Thailand

${ }^{3}$ Center of Excellence in Cardiac Electrophysiology Research, Chiang Mai University, Chiang Mai, Thailand ${ }^{4}$ Department of Oral Biology and Diagnostic Sciences, Faculty of Dentistry, Chiang Mai University, Chiang Mai, Thailand

Correspondence should be addressed to $\mathrm{N}$ Chattipakorn Email

nchattip@gmail.com

\begin{abstract}
Long-term high-fat diet (HFD) consumption causes cardiac dysfunction. Although calorie restriction (CR) has been shown to be useful in obesity, we hypothesized that combined CR with dipeptidyl peptidase-4 (DPP-4) inhibitor provides greater efficacy than monotherapy in attenuating cardiac dysfunction and metabolic impairment in HFDinduced obese-insulin resistant rats. Thirty male Wistar rats were divided into 2 groups to be fed on either a normal diet (ND, $n=6)$ or a HFD $(n=24)$ for 12 weeks. Then, HFD rats were divided into 4 subgroups ( $n=6 /$ subgroup) to receive just the vehicle, CR diet ( $60 \%$ of mean energy intake and changed to ND), vildagliptin ( $3 \mathrm{mg} / \mathrm{kg} /$ day) or combined $\mathrm{CR}$ and vildagliptin for 4 weeks. Metabolic parameters, heart rate variability (HRV), cardiac mitochondrial function, left ventricular (LV) and fibroblast growth factor (FGF) 21 signaling pathway were determined. Rats on a HFD developed insulin and FGF21 resistance, oxidative stress, cardiac mitochondrial dysfunction and impaired LV function. Rats on CR alone showed both decreased body weight and visceral fat accumulation, whereas vildagliptin did not alter these parameters. Rats in $C R$, vildagliptin and $C R$ plus vildagliptin subgroups had improved insulin sensitivity and oxidative stress. However, vildagliptin improved heart rate variability (HRV), cardiac mitochondrial function and LV function better than the CR. Chronic HFD consumption leads to obese-insulin resistance and FGF21 resistance. Although CR is effective in improving metabolic regulation, vildagliptin provides greater efficacy in preventing cardiac dysfunction by improving anti-apoptosis and FGF21 signaling pathways and attenuating cardiac mitochondrial dysfunction in obese-insulin-resistant rats.
\end{abstract}
Key Words
- caloric restriction
- obesity
- insulin resistance
- mitochondria
- FGF21 sensitivity
- apoptosis
- fatty acid $\beta$-oxidation
- cardiac function
- DPP-4 inhibitor

Journal of Endocrinology (2017) 232, 189-204

\section{Introduction}

Long-term consumption of high-fat diet (HFD) can lead to obese-insulin resistance (Pratchayasakul et al. 2011, Pipatpiboon et al. 2012), which is characterized by body weight gain, hyperinsulinemia and euglycemia and is also associated with metabolic dysregulation and dyslipidemia (Pratchayasakul et al. 2011, Pipatpiboon et al. 2013,

Published by Bioscientifica Ltd 
Apaijai et al. 2014). Obese-insulin resistance impairs not only the metabolic parameters but also damages the cardiovascular system including an increased blood pressure and cardiac dysfunction in various models (Fontana et al. 2004, Dolinsky et al. 2010). In past decades, cardiac mitochondrial dysfunction had been proposed as a crucial mechanism of cardiac dysfunction in high-fat diet-induced obese-insulin-resistant models (Chinda et al. 2013, Apaijai et al. 2014). Therefore, the therapeutic approaches that can attenuate metabolic complications and cardiac mitochondrial dysfunction caused by obeseinsulin resistance may lead to improved cardiac function.

Currently, lifestyle modification, including caloric restriction (CR), is the first therapeutic approach for diabetic management. CR combined with anti-diabetic drugs has been shown to be effective for metabolic regulation and glycemic control in diabetes (Inzucchi et al. 2015). Clinical studies showed that CR, with or without exercise, improved glycemic control, metabolic function, increased life spans and also reduced cardiovascular disease (CVD) risk factors in Type 2 diabetes mellitus (T2DM) patients (Wycherley et al. 2008). Furthermore, in animal studies, CR also reduced body weight, core temperature, heart rate and motor activity in normal rats (Aydin \& Gordon 2013). However, the role of CR on the heart in obese-insulin-resistant rats by switching from HFD to normal diet (ND) form is still unknown.

Fibroblast growth factor 21 (FGF21) is the most recent candidate FGF and plays a major role in metabolic regulation and lipid and energy homeostasis in a pathological state (Kharitonenkov 2009, Chau et al. 2010, Itoh \& Ornitz 2011). The FGF21 signaling pathways have a protective role in several organs including the liver (Yu et al. 2015) and pancreas (Wente et al. 2006), and it can also have a direct effect on the heart by activating the receptor complex FGFR1c/ $\beta$-Klotho (Planavila et al. 2013, 2014, Patel et al. 2014, Zhang et al. 2015). Previous studies demonstrated that FGF21 plays a cardioprotective role in arteriosclerosis (Lu et al. 2010, Wu et al. 2014), diabetes cardiomyopathy (DCM) (Yan et al. 2015, Zhang et al. 2015), cardiac ischemia-reperfusion (I/R) injury (Cong et al. 2013, Liu et al. 2013), myocardial infarction (MI) (Liu et al. 2012, 2013, Joki et al. 2015) and cardiac hypertrophy (Planavila et al. 2013, 2014). Interestingly, an increase in FGF21 levels is associated with future cardiovascular risk in T2DM patients (Shen et al. 2013, Ong et al. 2015). The 'FGF21 resistance' concept in obesity (Fisher et al. 2010, Tan et al. 2011) and in the heart (Patel et al. 2014) has been proposed. In addition, therapeutic strategies that overcome this condition also need to be investigated, and these therapies are expected to improve FGF21 sensitivity contributing to the potential cardioprotection role (Tanajak et al. 2015). Interestingly, a clinical study in obese children demonstrated that a low-carbohydrate diet and a low-fat diet consumption for 2 months improved the metabolic profile and decreased the serum FGF21 levels, suggesting that this CR could improve FGF21 sensitivity (Wycherley et al. 2008).

Vildagliptin is an oral anti-diabetic drug that has been used to treat T2DM patients (Inzucchi et al. 2015). Previous studies demonstrated that vildagliptin exerted cardioprotective effects in addition to its glycemic control in obese-insulin-resistant (Apaijai et al. 2012, 2013), MI (Inthachai et al. 2015) and $\mathrm{I} / \mathrm{R}$ injury rat models (Apaijai et al. 2014). Although both CR and vildagliptin exert cardioprotective effects, the effects of long-term $\mathrm{CR}$, vildagliptin and the combined therapy on metabolic regulation, heart rate variability (HRV), cardiac function, cardiac mitochondrial function and FGF21 signaling pathways in HFD-induced obese-insulin-resistant rats have not been investigated.

In this study, we hypothesized that long-term CR and vildagliptin increase insulin sensitivity, attenuate FGF21 resistance, improve cardiac mitochondrial fatty acid oxidation (FAO) and reduce cardiac apoptosis and cardiac mitochondrial dysfunction, leading to improved cardiac function in HFD-induced obese-insulin resistant rats and that combined therapy exerts greater cardioprotection than a single regimen.

\section{Material and methods}

\section{Ethical approval}

All experiments in this study were approved by the Faculty of Medicine, Chiang Mai University Institutional Animal Care and Use Committee (Permit No. 31/2557), in compliance with NIH guidelines and in accordance with the ARRIVE guidelines for reporting experiments involving animals (Kilkenny et al. 2010).

\section{Animals and diet}

Thirty adult male Wistar rats weighing 180-200g were obtained from the National Animal Center, Salaya Campus, Mahidol University, Bangkok, Thailand. All rats were housed in an environment with controlled temperature and humidity and a light-darkness (12:12 h) cycle. Rats were fed on either a ND or HFD. The ND is a standard laboratory pelleted diet (Mouse Feed Food

Published by Bioscientifica Ltd 
No. 082, C.P. Company, Bangkok, Thailand) containing $19.77 \%$ energy from fat and gives a total energy of $4.02 \mathrm{kcal} / \mathrm{g}$. The HFD contains $59.28 \%$ energy from fat and consists of standard rat diet (Mouse Feed Food No. 082, C.P. Company, Bangkok, Thailand, $365 \mathrm{~g} / \mathrm{kg}$ food), casein $(250 \mathrm{~g} / \mathrm{kg}$ food), lard $(310 \mathrm{~g} / \mathrm{kg}$ food), cholesterol $(10 \mathrm{~g} / \mathrm{kg}$ food), vitamins $(60 \mathrm{~g} / \mathrm{kg}$ food), DL-Methionine $(3 \mathrm{~g} / \mathrm{kg}$ food), yeast powder $(1 \mathrm{~g} / \mathrm{kg}$ food) and sodium chloride $(1 \mathrm{~g} / \mathrm{kg}$ food) giving a total energy of $5.35 \mathrm{kcal} / \mathrm{g}$ (Pratchayasakul et al. 2011). The CR was achieved in rats by switching from HFD to ND and also by decreasing the energy intake, after the 12-week average food intake, to $60 \%$ energy intake in ND form and feeding for 30 days (Wilsey \& Scarpace 2004).

\section{Drugs and vehicle}

The DDP-4 inhibitor vildagliptin ( $3 \mathrm{mg} / \mathrm{kg} / \mathrm{day}$ ) (Galvus, Novartis, Bangkok, Thailand) was used in this study. It was dissolved into $0.9 \%$ normal saline solution $(0.9 \%$ NSS) (Burkey et al. 2005, Apaijai et al. 2012, 2013). The $0.9 \%$ NSS in an equal volume was used as a vehicle (Apaijai et al. 2012, 2014). Rats were fed vildagliptin or the vehicle via oral gavage feeding once a day for 30 days.

\section{The experiments and study protocol}

After the acclimatization week, rats were divided into 2 groups and were given either ND $(n=6)$ or HFD $(n=24)$ for 12 weeks. At week 12, ND rats were continuously fed with ND and vehicle (NDV) for 30 days. For HFD rats, at week 12 , they were divided into 4 subgroups $(n=6 /$ group) and each group received one of the following: (1) vehicle (HFV); (2) CR diet and vehicle (HFCR); (3) vildagliptin (HFVil) and (4) CR diet plus vildagliptin (HFCRVil). All treatments were continued for 30 days. On week 12 and at the end of the experiments, rats in all groups were examined. The data collected were body weight, food intake, heart rate variability (HRV), cardiac function by echocardiography and tail blood pressure (BP) measurement using a tail cuff. In addition, the rats were fasted for $6 \mathrm{~h}$, and then blood was collected from the tail vein to determine the plasma metabolic parameters (fasting blood glucose (FPG), insulin, homeostasis model assessment (HOMA), total cholesterol (TC), triglyceride (TG), high-density lipoprotein cholesterol (HDL-C) and low-density lipoprotein cholesterol (LDL-C)) and plasma FGF21 by ELISA kit (R\&D Systems), and serum malondialdehyde (MDA) levels by high-performance liquid chromatography (HPLC) base assay. In addition, rats were fasted overnight to determine the insulin sensitivity using the oral glucose tolerance test (OGTT) (Apaijai et al. 2012, 2013). In addition, rats were fasted overnight to determine the insulin sensitivity using the oral glucose tolerance test (OGTT). An OGTT was carried out consisting of $2 \mathrm{~g} / \mathrm{kg}$ body weight glucose fed through a gavage (Apaijai et al. 2012, 2013). Blood was centrifuged at $3300 \mathrm{~g}, 4^{\circ} \mathrm{C}$ for $5 \mathrm{~min}$ for plasma collection and $825 \mathrm{~g}$, $4^{\circ} \mathrm{C}$ for $5 \mathrm{~min}$ for serum collection, then plasma and serum were kept at $-80^{\circ} \mathrm{C}$ until required (Thummasorn et al. 2011, Apaijai et al. 2012, 2013, Pipatpiboon et al. 2012, 2013, Surinkaew et al. 2013, Chinda et al. 2014). At the end of the experiments, rats had their left ventricular (LV) function determined by pressure-volume (P-V) loop measurement, and they were then killed to allow the following to be studied: cardiac mitochondrial function, cardiac mitochondrial morphology by transmission electron microscopy (TEM), cardiac tissue MDA levels by HPLC base assay and cardiac FGF21 signaling cascade protein expression by Western blot analysis. Moreover, the visceral fat was weighed on removal from the rats after killing. The experimental protocol of this study is shown in Fig. 1.

\section{Metabolic parameters assessments}

A commercial colorimetric assay kit (Erba Diagnostics Mannheim GmbH, Mannheim, Germany) was purchased for the determination of FPG, TC and TG levels (Pratchayasakul et al. 2011, Apaijai et al. 2014). A second commercial colorimetric assay kit (Biovision) was purchased for determining the plasma HDL-C level (Apaijai et al. 2013). After that the Friedewald equation was used to calculate the plasma LDL-C levels (Friedewald et al. 1972, Apaijai et al. 2014). The commercial sandwich ELISA kit (LINCO Research) was purchased to determine the plasma insulin levels (Apaijai et al. 2013, 2014). The degree of insulin sensitivity was determined by using the HOMA index, an increase in the HOMA index indicates a higher degree of insulin resistance (Pipatpiboon et al. 2012).

\section{Plasma FGF21 assessment}

Plasma FGF21 levels were determined using a quantitative sandwich enzyme immunoassay technique by using a mouse/Rat FGF21 ELISA kit (R\&D Systems) (Yan et al. 2015).

Published by Bioscientifica Ltd 


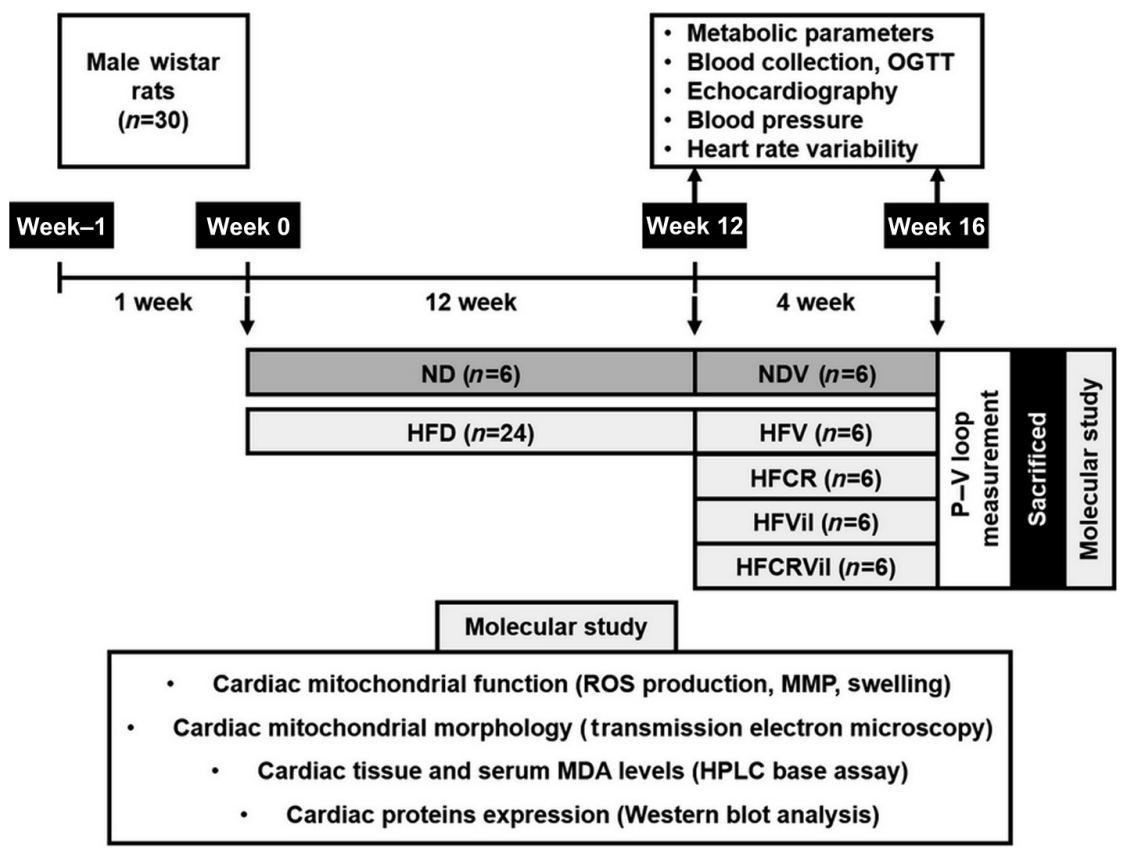

\section{Figure 1}

The experimental protocol of the study. HFCR, high-fat diet treated with CR diet; HFCRVil, high-fat diet treated with $C R$ diet and vildagliptin; HFD, high-fat diet; HFV, high-fat diet treated with vehicle; HFVil, high-fat diet treated with vildagliptin; HPLC, high-performance liquid chromatography; MDA, Malondialdehyde; MMP, mitochondrial membrane potential; ND, normal diet; NDV, normal diet treated with vehicle; OGTT, oral glucose tolerance test; P-V loop, pressurevolume loop; ROS, reactive oxygen species.

\section{Heart rate variability (HRV) determination}

Electrocardiography was performed by immobilizing the limbs of rats in a prone position under $2.5 \%$ isoflurane inhalation anesthesia. Needle electrodes were inserted subcutaneously into the positions of lead II electrocardiogram (ECG). Rats were allowed to gain full consciousness prior to ECG recording. During ECG recording, rats were kept restrained and prohibited from movement. HRV was determined from lead II ECG by using PowerLab (AD Instruments, Colorado Springs, CO, USA) equipped with the Chart 5.0 program for $20 \mathrm{~min}$. A stableECG wasused to determine the relationshipbetween the RR interval and the beat numbers (Tachogram) using a MATLAB program (Chattipakorn et al. 2007, Pratchayasakul et al. 2011, Apaijai et al. 2013). Power spectra of RR interval variability were obtained using the fast Fourier transform (FFT) algorithm. The high frequency (HF; $0.6-3 \mathrm{~Hz}$ ) band, low frequency (LF; $0.2-0.6 \mathrm{~Hz}$ ) band and very low frequency (VLF; below $0.2 \mathrm{~Hz}$ ) band were detected. Each spectral component was calculated as intervals under the respective part of the power spectral density function and was presented in absolute units $\left(\mathrm{ms}^{2}\right)$ (Chattipakorn et al. 2007). To minimize the effect of changes in total power on the LF and HF bands, LF and HF were expressed as normalized units by dividing the reading by the total power minus VLF (Chattipakorn et al. 2007). The increase of the LF/HF ratio indicates an increase in sympathetic activity or depressed HRV (Pongchaidecha et al. 2009, Apaijai et al. 2013).

\section{Tail cuff blood pressure measurement}

Blood pressure was measured by using the non-invasive CODA 2 on the volume-pressure recording (VPR) tailcuff method (Kent Scientific Corporation, Torrington, CT, USA) (Feng et al. 2008). Rat tails were attached to an occlusion cuff proximally (O-Cuff) and VPR distally and acclimatized in the holder on the infrared warming platform $32-35^{\circ} \mathrm{C}$ using levels for this temperature control for at least $5 \mathrm{~min}$. The first five cycles were acclimatization cycles and were not included in the data analysis. The mean blood pressure was calculated from the next 20 consecutive cycles.

\section{LV function study for echocardiography}

Rats were anesthetized by inhaling isoflurane and oxygen supplementation at 3 liters per minute for 3-5 min. During anesthesia, the LV function was determined using a Vivid i echocardiograph (GE Medical Systems). The image by echocardiography was produced by M-mode and collected in the parasternal short axis of the heart at the papillary muscle levels and \% fractional shortening (\%FS) was determined. Increased \%FS indicated increased LV contractile function (Apaijai et al. 2012, 2013). 


\section{LV function using a P-V loop}

Rats were anesthetized by intramuscular injection with Zoletil (50 mg/kg, Vibbac Laboratories, Carros, France) plus Xylazine $(0.15 \mathrm{mg} / \mathrm{kg}$, Laboratories Calier, S.A., Barcelona, Spain). After that an incision was made on the midline of the anterior cervical area and then a tracheostomy tube was inserted into the trachea. Subsequently, the right common carotid artery (CCA) was identified and the P-V loop catheter (Scisense, Ontorio, Cannada) was inserted into the right CCA and placed into the ascending aorta allowing the recording of systolic blood pressure (SBP) and diastolic blood pressure (DBP). Then, the catheter was advanced into the LV chamber for determining the LV function including heart rate (HR), end-systolic pressure (ESP), end-diastolic pressure (EDP), $d P / d t_{\max }, d P / d t_{\min }$ and stroke volume (SV). Data from the $\mathrm{P}-\mathrm{V}$ loop measurement were recorded using an analytical software program (Labscribe2, Dover, New Hampshire, USA) (Apaijai et al. 2013, Chinda et al. 2014). At the end of this protocol, the rats were decapitated, and the hearts were removed rapidly for further cardiac tissue protocols described below.

\section{Determination of cardiac mitochondrial function and morphology}

The rats hearts were perfused with $10 \mathrm{~mL}$ of $0.9 \%$ NSS and removed rapidly. The cardiac mitochondrial isolation protocols were performed as previously described (Thummasorn et al. 2011, Apaijai et al. 2012, 2013). The cardiac mitochondria reactive oxygen species (ROS) production was determined by incubating the mitochondria with $2 \mathrm{M}$ DCFH-DA dye at $25^{\circ} \mathrm{C}$ for $20 \mathrm{~min}$, and the fluorescent intensity of the solution was detected by a fluorescent microplate reader with excitation wavelength at $485 \mathrm{~nm}$ and emission wavelength at $530 \mathrm{~nm}$ (BioTek Instruments). An increase in the fluorescent intensity indicated an increased mitochondrial ROS production (Thummasorn et al. 2011, Chinda et al. 2013).

The cardiac mitochondrial membrane potential change $(\Delta \Psi)$ was determined using $5-\mu \mathrm{M}$ JC-1 dye and incubating the mitochondria with $\mathrm{JC}-1$ at $37^{\circ} \mathrm{C}$ for $30 \mathrm{~min}$ (Thummasorn et al. 2011, Apaijai et al. 2014). The cardiac mitochondrial membrane potential changes were detected using a fluorescent microplate reader. The JC-1 monomer form concentration is represented by the green fluorescence and is excited by a wavelength of $485 \mathrm{~nm}$ and the emission is detected at $590 \mathrm{~nm}$. The aggregate form of
JC-1 is represented by the red fluorescence and is excited at a wavelength of $485 \mathrm{~nm}$ and the emission is detected at $530 \mathrm{~nm}$. A decrease in the red/green fluorescent intensity ratio indicates an increase in cardiac mitochondrial membrane depolarization (Thummasorn et al. 2011, Chinda et al. 2013).

The cardiac mitochondrial swelling was determined by incubating cardiac mitochondria with $1.5-\mathrm{mM}$ respiration buffer. The absorbance was determined using a spectrophotometer. A decreased absorbance indicated increased cardiac mitochondrial swelling (Thummasorn et al. 2011, Apaijai et al. 2013).

The cardiac mitochondria were collected during the cardiac mitochondrial function assessment and fixed by $2.5 \%$ glutaraldehyde in a $0.1-\mathrm{M}$ phosphate buffer overnight. After that, they were fixed in a $1 \%$ cacodylatebuffer osmium tetroxide for $2 \mathrm{~h}$ and a graded series of ethanol were used to dehydrate them. A diamond knife was used to cut the cardiac mitochondria embedded in Epon-Araldite and stained with uranyl acetate. Finally, the cardiac mitochondria's morphology was observed using a transmission electron microscope (TEM) at 15,000× by JEM-2200FS field emission electron microscope (Thummasorn et al. 2011, Apaijai et al. 2014).

\section{Determination of MDA concentration}

The cardiac tissue and plasma MDA concentrations were determined using a HPLC-based assay (Thermo Scientific) (Apaijai et al. 2012, 2013). Plasma and cardiac MDA were mixed with $\mathrm{H}_{3} \mathrm{PO}_{4}$ and thiobarbituric acid (TBA) to produce TBA-reactive substances (TBARS). The plasma and cardiac TBARS concentration were determined directly from a standard curve and reported as equivalent to the MDA concentration (Apaijai et al. 2012, 2013).

\section{Cardiac protein expression by Western blot analysis}

Cardiac tissues for determining the protein expressions were obtained from the fresh hearts at the LV apex. Myocardial protein extracts were prepared by homogenization of nitrogen-frozen myocardial tissues in a 300- $\mu \mathrm{L}$ extraction buffer containing 20-mM Tris$\mathrm{HCl}$ ( $\mathrm{pH}$ 6.8), 1-mM sodium orthovanadate, 5-mM sodium fluoride and protease inhibitor. Total protein concentration was determined using a Bio-Rad protein assay kit (Bio-Rad Laboratories). Total protein was mixed with loading buffer (5\% beta mercaptoethanol, 0.05\% bromophenol blue, $75 \mathrm{mM}$ Tris- $\mathrm{HCl}$ (pH 6.8), 2\% SDS 
Table 1 The effects of calorie restriction, vildagliptin and calorie restriction plus vildagliptin on metabolic parameters in HFD rats after treatment for 4 weeks. Body weight and visceral fat were recorded at the end of experiment. Moreover, the rats were fasted for $6 \mathrm{~h}$ then blood was collected from the tail vein to determine the plasma metabolic parameters (fasting plasma glucose FBS, insulin, Homeostasis Model Assessment (HOMA)-index, total cholesterol (TC), triglyceride (TG), high density lipoproteincholesterol (HDL-C), and low-density lipoprotein-cholesterol (LDL-C), serum maladehyde (MDA) levels and cardiac tissue MDA by high liquid chromatography ( $\mathrm{HPL}) \mathrm{C}$ base assay. In addition, rats were fasted overnight to determine the insulin sensitivity using the area under the curve (AUCg) by oral glucose tolerance test (OGTT).

\begin{tabular}{|c|c|c|c|c|c|}
\hline \multirow[b]{2}{*}{ Parameters } & \multicolumn{5}{|c|}{ Groups } \\
\hline & NDV & HFV & HFCR & HFVil & HFCRVil \\
\hline Body weight (g) & $519 \pm 24$ & $627 \pm 21 *$ & $523 \pm 23^{\dagger}$ & $625 \pm 38^{*, \neq}$ & $518 \pm 14^{\dagger, \#}$ \\
\hline Visceral fat (g) & $32 \pm 3$ & $54 \pm 4 *$ & $36 \pm 4^{\dagger}$ & $58 \pm 5^{*, \neq}$ & $30 \pm 2^{+, \#}$ \\
\hline Plasma glucose (mM) & $8.1 \pm 1.0$ & $8.4 \pm 0.2$ & $8.5 \pm 0.1$ & $8.5 \pm 0.3$ & $8.6 \pm 0.3$ \\
\hline Plasma TC (mM) & $3.9 \pm 0.3$ & $5.4 \pm 0.2 *$ & $4.2 \pm 0.1^{\dagger}$ & $4.3 \pm 0.5^{+}$ & $4.2 \pm 0.3^{\dagger}$ \\
\hline Plasma TG (mM) & $2.9 \pm 0.5$ & $3.1 \pm 0.3$ & $2.8 \pm 0.3$ & $2.7 \pm 0.2$ & $2.8 \pm 0.4$ \\
\hline Plasma HDL-C (mM) & $1.9 \pm 0.1$ & $1.4 \pm 0.1 *$ & $2.1 \pm 0.1^{\dagger}$ & $1.9 \pm 0.1^{\dagger}$ & $2.0 \pm 0.1^{\dagger}$ \\
\hline Plasma LDL-C (mM) & $1.3 \pm 0.1$ & $3.0 \pm 0.4^{*}$ & $1.6 \pm 0.4^{\dagger}$ & $1.4 \pm 0.2^{+}$ & $1.4 \pm 0.2^{\dagger}$ \\
\hline Plasma insulin ( $\mathrm{ng} / \mathrm{mL})$ & $4.3 \pm 0.3$ & $6.7 \pm 0.8 *$ & $4.1 \pm 0.5^{\dagger}$ & $3.8 \pm 0.5^{\dagger}$ & $4.6 \pm 0.6^{\dagger}$ \\
\hline HOMA index & $33.2 \pm 6.1$ & $61.5 \pm 10.0 *$ & $33.6 \pm 5.1^{\dagger}$ & $29.8 \pm 3.8^{\dagger}$ & $33.8 \pm 7.2^{\dagger}$ \\
\hline Serum MDA $(\mu \mathrm{mol} / \mathrm{mL})$ & $1.5 \pm 0.1$ & $6.0 \pm 1.4^{*}$ & $1.7 \pm 0.1^{\dagger}$ & $1.2 \pm 0.1^{\dagger}$ & $1.5 \pm 0.1^{\dagger}$ \\
\hline $\begin{array}{l}\text { Tissue MDA ( } \mu \mathrm{mol} / \mathrm{mg} \\
\text { protein) }\end{array}$ & $0.80 \pm 0.08$ & $2.91 \pm 0.54^{*}$ & $0.71 \pm 0.08^{\dagger}$ & $0.64 \pm 0.07^{\dagger}$ & $0.72 \pm 0.06^{+}$ \\
\hline AUCg $\left(\mathrm{mM} \times \min \times 10^{5}\right)$ & $9.23 \pm 0.25$ & $12.6 \pm 0.70 *$ & $8.87 \pm 0.57^{\dagger}$ & $9.81 \pm 0.27^{\dagger}$ & $9.08 \pm 0.32^{\dagger}$ \\
\hline
\end{tabular}

$\star P<0.05$ vs NDV, ${ }^{\dagger} P<0.05$ vs HFV, ${ }^{*} P<0.05$ vs HFCR.

HFCR, high-fat diet treated with CR diet; HFCRVil, high-fat diet treated with CR diet and vildagliptin; HFD, high-fat diet; HFV, high-fat diet treated with vehicle; HFVil, high-fat diet treated with vildagliptin; ND, normal diet; NDV, normal diet treated with vehicle.

and $10 \%$ glycerol) and boiled at $95^{\circ} \mathrm{C}$ for $10 \mathrm{~min}$. Forty micrograms of total proteins from each group were loaded in each well (total volume was $20 \mu \mathrm{L} /$ well). The samples were loaded onto the $10 \%$ SDS-polyacrylamide gels (Apaijai et al. 2014, Pongkan et al. 2016). Then, proteins were transferred to nitrocellulose membranes in a glycine/methanol transfer buffer in a wet/tank blotting system (Bio-Rad Laboratories). Membranes were blocked in either $5 \%$ skim milk or $5 \%$ bovine serum albumin in Tris-buffered saline and Tween (TBST) buffer. The FGF21 signaling proteins including fibroblast growth factor receptor (FGFR) 1 and phospho-FGFR1 (p-FGFR1) (1:200 dilution, Sigma-Aldrich), $\quad \beta$-Klotho $\quad(1: 200$ dilution), extracellular signal-regulated kinase (ERK1/2), phosphor-ERK1/2 (p-ERK1/2) (1:1000 dilution, Santa Cruz technologies), Akt and p-Akt (1:500 dilution, Cell Signaling Technology) were determined. Moreover, Bax (1:1000 dilution), cleaved caspase-3 (1:1000 dilution), proliferator-activated receptor gamma coactivator 1-alpha (PGC-1 $\alpha$ ) (1:200 dilution), carnitine palmitoyltransferase 1 (CPT1) (1:200 dilution), $\beta$-actin (1:2000 dilution, Santa Cruz Biotechnology) and B-cell lymphoma 2 (Bcl2) (1:1000 dilution, Cell Signaling Technology) were determined. Bound antibody for $\beta$-actin (Surinkaew et al. 2013) was detected by horse anti-mouse IgG-conjugated horseradish peroxidase (HRP)-linked antibody (1:2000 dilution, Cell Signaling Technology). Bound antibody for $\beta$-Klotho was detected using a rabbit anti-goat IgGconjugated HRP-linked antibody (1:2000 dilution, Santa Cruz Biotechnology). Bound antibodies for FGFR1, p-FGFR1, ERK1/2, p-ERK1/2, Akt, p-Akt, Bax, Bcl-2, cleaved caspase-3, PGC- $1 \alpha$ and CPT-1 were detected using a goat anti-rabbit IgG-conjugated HRP-linked antibody (1:2000 dilution, Cell Signaling Technology). Enhanced chemiluminescence (ECL) detection reagents were used to visualize peroxidase reaction products (Clarity ECL Western blotting substrate, Bio-Rad) (Pongkan et al. 2016). However, due to our protocol, we used invisible markers BLUeye Prestained Protein Ladder (Cat. No. PM007-0500; GeneDireX, Inc. Miaoli County 350, Taiwan, R.O.C.). The WesternSure Pen (Li-cor biotechnology, Lincoln, Nebraska, USA) was used to annotate visible protein ladders prior to detecting chemiluminescent intensity from Western blot using the ChemiDoc system. The membranes were developed in the ChemiDoc touch imaging system (Bio-Rad Laboratories) by placing a membrane on the chemi/UV/strain-free tray. Then, the image was adjusted to an appropriate size, and the blot 
Table 2 The effects of calorie restriction, vildagliptin, and calorie restriction plus vildagliptin on heart rate and blood pressure in HFD rats after treatment for 4 weeks. At the end of experiment, systolic blood pressure (SBP), diastolic blood pressure (DBP), mean arterial pressure (MAP), and heart rate (HR) were was measured by using the non-invasive CODA 2 on the volume-pressure recording (VPR) tail-cuff method.

\begin{tabular}{|c|c|c|c|c|c|}
\hline \multirow[b]{2}{*}{ Parameters } & \multicolumn{5}{|c|}{ Groups } \\
\hline & NDV & HFV & HFCR & HFVil & HFCRVil \\
\hline $\mathrm{SBP}(\mathrm{mmHg})$ & $132 \pm 5$ & $156 \pm 3 *$ & $152 \pm 8 *$ & $134 \pm 4^{+\neq}$ & $129 \pm 5^{\dagger, \ddagger}$ \\
\hline $\mathrm{DBP}(\mathrm{mmHg})$ & $87 \pm 7$ & $88 \pm 3$ & $87 \pm 6$ & $82 \pm 5$ & $87 \pm 6$ \\
\hline MAP (mmHg) & $104 \pm 4$ & $117 \pm 2 *$ & $118 \pm 3 *$ & $103 \pm 3^{\dagger, \ddagger}$ & $100 \pm 3^{\dagger, \ddagger}$ \\
\hline HR (bpm) & $401 \pm 6$ & $436 \pm 10 *$ & $429 \pm 10 *$ & $383 \pm 21^{\dagger, \neq}$ & $392 \pm 12^{\dagger, \ddagger}$ \\
\hline
\end{tabular}

$* P<0.05$ vs NDV, $+P<0.05$ vs HFV, $\neq P<0.05$ vs HFCR.

HFCR, high-fat diet treated with CR diet; HFCRVil, high-fat diet treated with CR diet and vildagliptin; HFD, high-fat diet; HFV, high-fat diet treated with vehicle; HFVil, high-fat diet treated with vildagliptin; ND, normal diet; NDV, normal diet treated with vehicle.

chemiluminescence category was chosen for exposure and to take a Western blot image. The densitometric analysis was done by the ImageJ program (Chinda et al. 2014). Each protein expression was normalized with $\beta$-actin expression.

\section{Statistical analysis}

Data were expressed as mean \pm S.E.M. One-way analysis of variance (ANOVA) by least significant difference (LSD) post hoc test was used to test the difference between the groups. $P<0.05$ was considered statistically significant.

\section{Results}

\section{$C R$, vildagliptin and combined therapy restored metabolic disturbance in obese-insulin-resistant rats}

At 16 weeks after HFD feeding, HFV rats had significantly increased BW and visceral fat, compared with the NDV group (Table 1). The dietary intervention HFCR group had significantly decreased BW and visceral fat, compared with the HFV group. However, no differences in the BW and visceral fat were found in the HFVil rats, compared with the HFV group. Nevertheless, the combined therapy HFCRVil rats had significantly decreased BW and visceral fat, compared with the HFV and HFVil groups (Table 1).

The plasma TC, plasma LDL-C, plasma insulin, HOMA index, serum MDA, cardiac MDA, AUCg and the HOMA index were increased, whereas the plasma HDL-C was decreased in the HFV rats, compared with the NDV group (Table 1). These parameters were improved in the HFCR, HFVil and HFCRVil rats, when compared with the HFV group. However, plasma glucose and plasma TG showed no difference between the groups $(P>0.05$, Table 1$)$. Moreover, the HFV rats had significantly increased HR, SBP and MAP, compared with the NDV group. Only rats in the vildagliptin-treated groups (HFVil and HFCRVil) had significantly decreased HR, SBP and MAP, compared with the HFV group (Table 2).

\section{Vildagliptin increased cardiac autonomic regulation and LV function in obese-insulin-resistant rats more extensively than $\mathrm{CR}$}

Regarding HRV, the results showed that HFD results showed a significant increase in the LF/HF ratio since week 12 in the HFD rats, compared with the ND group (Fig. 2A). In the same way, it was found that the \%FS was significantly decreased at week 12 in the HFD group, compared with the ND group (Fig. 2B). After 16 weeks of HFD feeding, there was still a significant increase in the LF/HF ratio and decreased \%FS in the HFV rats, compared with the NDV group. In addition, the LF/HF ratio was significantly decreased in the HFCR group (Fig. 2C), when compared with the HFV group. Moreover, the LF/HF ratio was also significantly decreased in the HFVil and HFCRVil groups (Fig. 2C), when compared with the HFV and HFCR groups. Furthermore, the \%FS was significantly increased in the HFCR group (Fig. 2D), when compared with the $\mathrm{HFV}$ group. Moreover, the \%FS was also significantly increased in the HFVil and HFCRVil groups (Fig. 2D), when compared with the HFV and HFCR groups.

At 16 weeks after HFD feeding, the P-V loop showed that the HFV rats had significantly increased SBP and MAP and also had impaired cardiac function identified by increased HR and EDP, and decreased ESP, $\pm d P / d t$ http://joe.endocrinology-journals.org DOI: $10.1530 / J O E-16-0406$ (c) 2017 Society for Endocrinology Printed in Great Britain
Published by Bioscientifica Ltd 

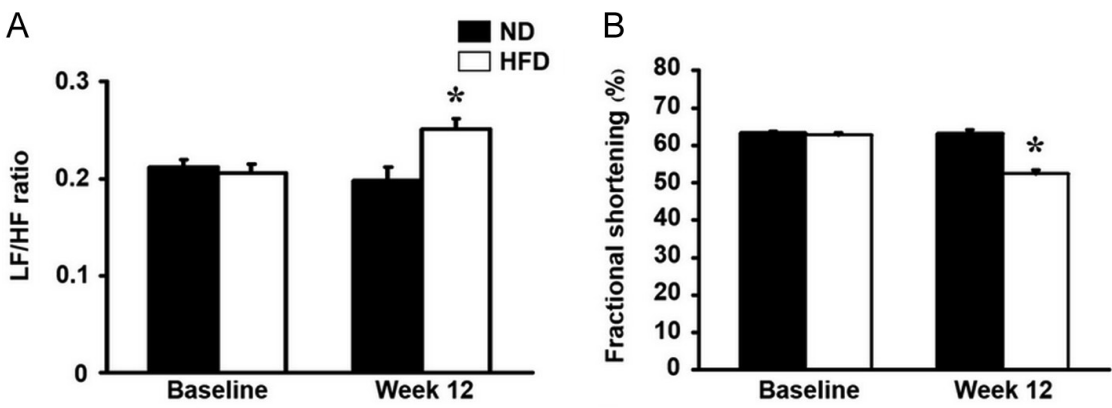

\section{Figure 2}

The effects of HFD consumption, HFCR, vildagliptin, and combined HFCR and vildagliptin on $\mathrm{HRV}$ and echocardiographic parameters. (A) HRV and (B) \% fractional shortening (\%FS) in ND and HFD rats at baseline and week $12 .{ }^{*} P<0.05$ vs ND (independent sample $t$-test, $n=6$ for ND group
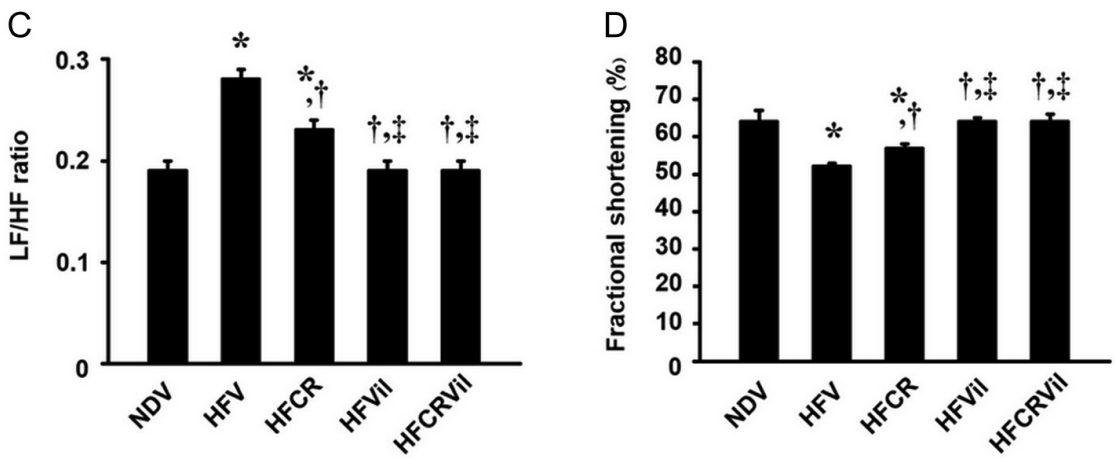
and $n=12$ for HFD group). (C) HRV and (D) \%FS in ND and HFD rats treated with vehicle, calorie restriction (CR), vildagliptin and CR combined vildagliptin. ${ }^{*} P<0.05$ vs NDV, ${ }^{+} P<0.05$ vs HFV, ${ }^{\ddagger} P<0.05$ vs HFCR, ${ }^{P} P<0.05$ vs HFCR (1-WAY ANOVA, LSD post hoc test, $n=6$ per subgroups). HFCR, high-fat diet treated with CR diet; HFCRVil, high-fat diet treated with $C R$ diet and vildagliptin; HFD, high-fat diet; HF, high frequency; HFV, high-fat diet treated with vehicle; HFVil, high-fat diet treated with vildagliptin; HRV, heart rate variability; LF, low frequency; ND, normal diet; NDV, normal diet treated with vehicle.

and SV, when compared with the NDV group (Table 3). However, only rats in the vildagliptin-treated groups (HFVil and HFCRVil) had improved blood pressure indicated by decreased SBP and MAP, when compared with the HFV group. The HFCR group had improved cardiac function shown by increased ESP, $\pm d P / d t$ and SV and also decreased HR and EDP, when compared with the HFV group (Table 3). Moreover, vildagliptin-treated groups (HFVil and HFCRVil) also had improved cardiac function illustrated by increased ESP, $\pm d P / d t$ and SV, and also decreased HR and EDP, when compared with the HFV and HFCR groups (Table 3).

\section{Vildagliptin restored FGF21 sensitivity and activated anti-apoptotic pathways in the heart more effectively than CR}

The levels of plasma FGF21 were significantly higher in the HFV group, compared to the ND rats at week 12 of HFD feeding (Fig. 3A). Plasma FGF21 levels at week 16 were also

Table 3 Effects of calorie restriction, vildagliptin, and calorie restriction plus vildagliptin on cardiac function in HFD rats after treatment for 4 weeks. At the end of experiment left ventricular (LV) function was measured by using the pressure-volume (P-V) loop. P-V loop catheter (Scisense, Ontorio, Cannada) was inserted into the right common carotid artery (CCA) and placed into the ascending aorta allowing the recording of systolic blood pressure (SBP) and diastolic blood pressure (DBP). Then the catheter was advanced into the LV chamber for the determining of LV function including heart rate (HR), end-systolic pressure (ESP), end-diastolic pressure (EDP), $d P / d t_{\max } d P / d t_{\min ^{\prime}}$ and stroke volume (SV).

\begin{tabular}{|c|c|c|c|c|c|}
\hline \multirow[b]{2}{*}{ Parameters } & \multicolumn{5}{|c|}{ Groups } \\
\hline & NDV & HFV & HFCR & HFVil & HFCRVil \\
\hline HR (bpm) & $303 \pm 26$ & $383 \pm 6 *$ & $370 \pm 2 *$ & $326 \pm 11^{t, \neq}$ & $324 \pm 2^{\dagger, \ddagger}$ \\
\hline $\mathrm{SBP}(\mathrm{mmHg})$ & $127 \pm 1$ & $135 \pm 1$ * & $133 \pm 1$ * & $125 \pm 2^{\dagger, \ddagger}$ & $125 \pm 1^{\dagger, \neq}$ \\
\hline $\mathrm{DBP}(\mathrm{mmHg})$ & $84 \pm 1$ & $84 \pm 1$ & $85 \pm 1$ & $84 \pm 1$ & $84 \pm 1$ \\
\hline ESP (mmHg) & $127 \pm 1$ & $106 \pm 1 *$ & $116 \pm 2 *,+$ & $125 \pm 3^{\dagger, \ddagger}$ & $124 \pm 1^{\dagger, \ddagger}$ \\
\hline EDP $(\mathrm{mmHg})$ & $19 \pm 1$ & $36 \pm 1 *$ & $26 \pm 1 *,+$ & $19 \pm 1^{t, \neq}$ & $19 \pm 1^{\dagger, \ddagger}$ \\
\hline$+d P / d t(\mathrm{mmHg} / \mathrm{s})$ & $8360 \pm 29$ & $6268 \pm 89 *$ & $7567 \pm 99 *,+$ & $8298 \pm 165^{\dagger, \neq}$ & $8341 \pm 50^{\dagger, \ddagger}$ \\
\hline$-d P / d t(\mathrm{mmHg} / \mathrm{s})$ & $5681 \pm 188$ & $3852 \pm 100 *$ & $5273 \pm 65^{*}++$ & $5595 \pm 39+, \neq$ & $5646 \pm 57^{\dagger, \ddagger}$ \\
\hline $\mathrm{SV}(\mathrm{mL} / \mathrm{g} \cdot \mathrm{BW})$ & $1.06 \pm 0.02$ & $0.75 \pm 0.02 *$ & $0.93 \pm 0.05 *, \dagger$ & $1.08 \pm 0.03^{\dagger, \ddagger}$ & $1.14 \pm 0.05^{\dagger, \ddagger}$ \\
\hline
\end{tabular}

${ }^{*} P<0.05$ vs NDV, ${ }^{+} P<0.05$ vs HFV, ${ }^{\ddagger} P<0.05$ vs HFCR.

HFCR, high-fat diet treated with CR diet; HFCRVil, high-fat diet treated with CR diet and vildagliptin; HFD, high-fat diet; HFV, high-fat diet treated with vehicle; HFVil, high-fat diet treated with vildagliptin; ND, normal diet; NDV, normal diet treated with vehicle.

http://joe.endocrinology-journals.org
DOI: $10.1530 / \mathrm{JOE}-16-0406$
○ 2017 Society for Endocrinology Printed in Great Britain
Published by Bioscientifica Ltd 
A

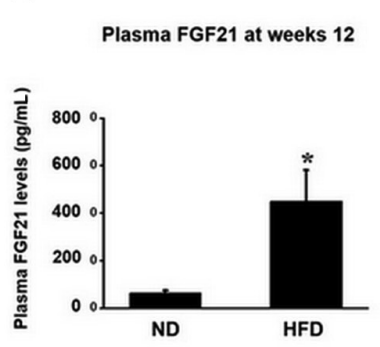

D

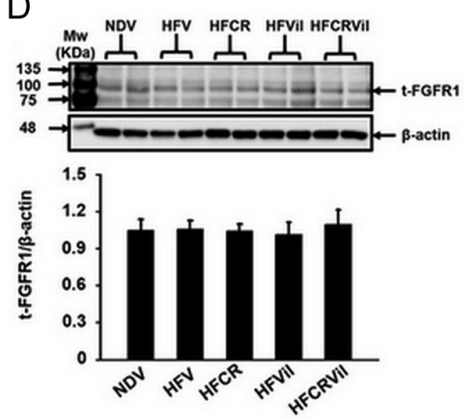

G
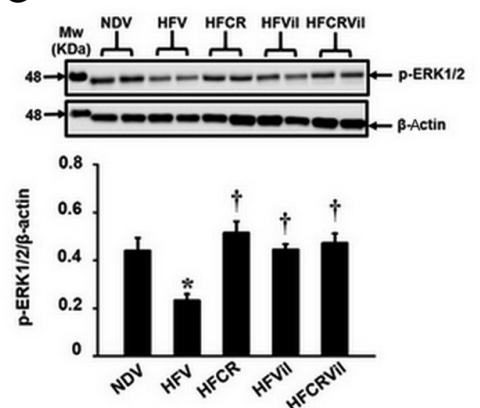

B

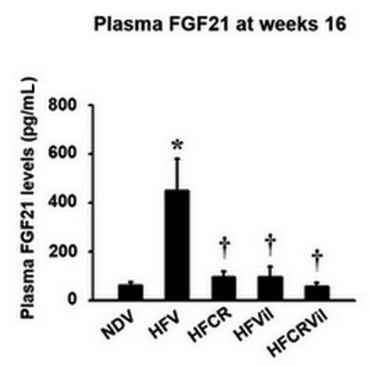

E

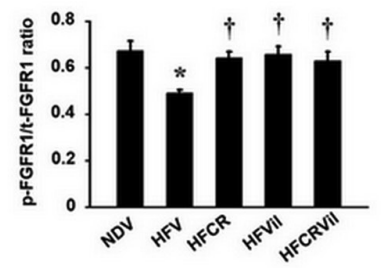

$\mathrm{H}$
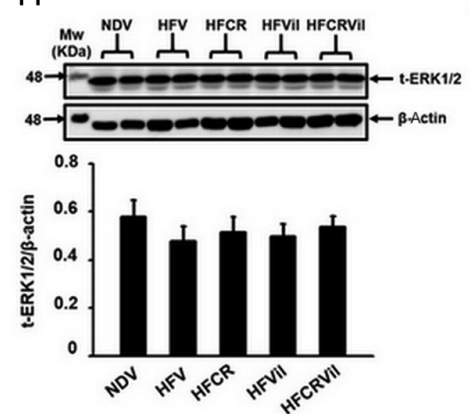

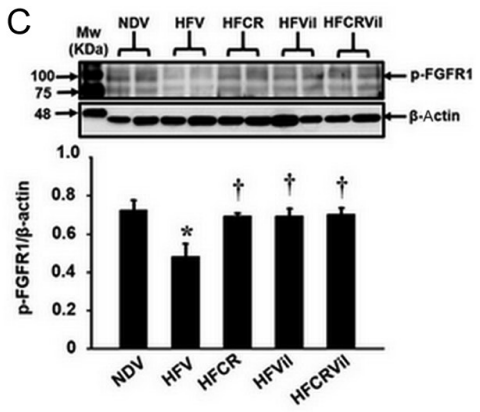

F
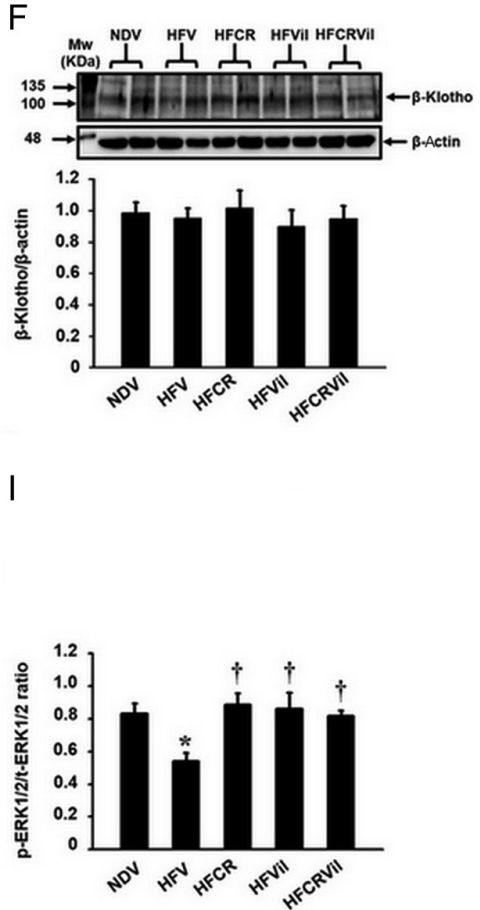

Figure 3

The effects of HFCR, vildagliptin and combined HFCR and vildagliptin on plasma FGF21 levels, FGFR1//-Klotho receptors complex and intracellular Erk1/2 expression and phosphorylation. (A) Plasma FGF21 levels at weeks 12, (B) plasma FGF21 levels post treatment, (C) p-FGFR1, (D) t-FGFR1, (E) p-FGFR1/t-FGFR1, (F) $\beta$-Klotho, (G) p-Erk1/2, (H) t-Erk1/2, and (I) p-Erk1/2/t-Erk1/2 ratio in ND and HFD rats treated with vehicle, Calorie restriction (CR), vildagliptin, and CR combined with vildagliptin. ${ }^{*} P<0.05$ vs NDV, ${ }^{+} P<0.05$ vs HFV, ${ }^{*} P<0.05$ vs HFCR (1-WAY ANOVA, LSD post hoc test, $n=4-6$ per subgroups). Erk1/2, extracellular signal-regulated protein kinases 1 and 2; FGFR1, fibroblast growth factor receptors 1; HFCR, high-fat diet treated with CR diet; HFCRVil, high-fat diet treated with CR diet and vildagliptin; HFD, high-fat diet; HFV, high-fat diet treated with vehicle; HFVil, high-fat diet treated with vildagliptin; ND, normal diet; NDV, normal diet treated with vehicle; p-FGFR1, phospho FGFR1; p-ERK1/2, phospho ERK1/2; t-ERK1/2, total ERK $1 / 2$.

significantly increased in the HFV group, compared with the NDV group (Fig. 3B). However, the plasma FGF21 levels were significantly decreased in the HFCR, HFVil and HFCRVil groups (Fig. 3B). The FGF21 receptor complex and signaling cascade protein expression and phosphorylation by Western blot analysis demonstrated that the HFV group had decreased p-FGFR1 (Fig. 3C) and also a p-FGFR1/ t-FGFR1 ratio (Fig. 3E) when compared with the NDV group. HFCR, HFVil and HFCRVil groups had restored p-FGFR1 (Fig. 3C) and p-FGFR1/t-FGFR1 ratios when compared with the HFV group (Fig. 3E). However, no alteration of t-FGFR1 (Fig. 3D) and $\beta$-Klotho (Fig. 3F) expression were found in all groups. Regarding downstream signaling, the HFV rats had decreased p-ERK1/2 (Fig. 3G) and also p-ERK1/2/tERK1/2 ratio (Fig. 3I), whereas all treatments increased p-ERK1/2 (Fig. 3G) and p-ERK1/2/t-ERK1/2 ratios (Fig. 3I). However, no alteration of t-ERK1/2 (Fig. 3H) expression was found in all groups. In addition, the p-Akt/t-Akt ratio was not significantly different between groups $(0.57 \pm 0.05$, $0.49 \pm 0.06,0.55 \pm 0.12,0.47 \pm 0.07$ and $0.51 \pm 0.10$ unit for NDV, HFV, HFCR, HFVil and HFCRVil, respectively).

In regard to anti-apoptotic signaling pathways, rats in the HFV group had significantly increased apoptotic protein expression including Bax (Fig. 4A), Bax/BCL-2

Published by Bioscientifica Ltd. 
A
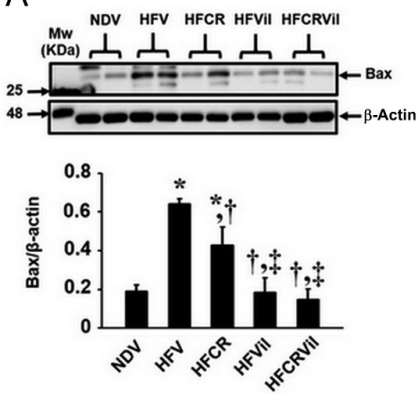

D NDV HFV HFCR HFVil HFCRViI
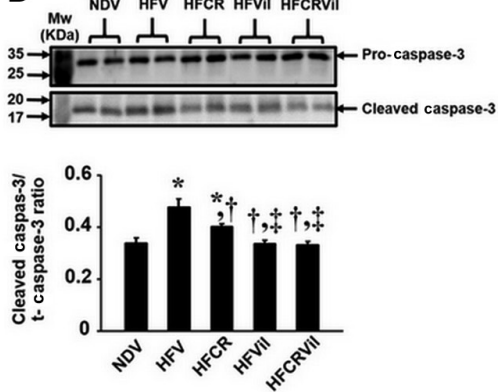

B
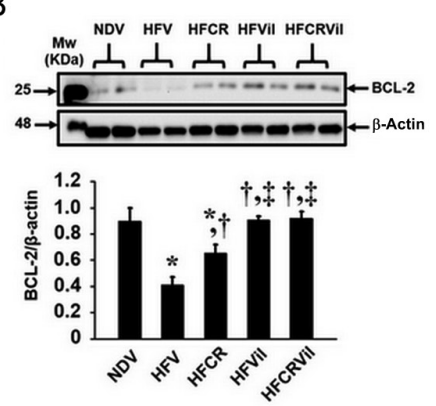

E
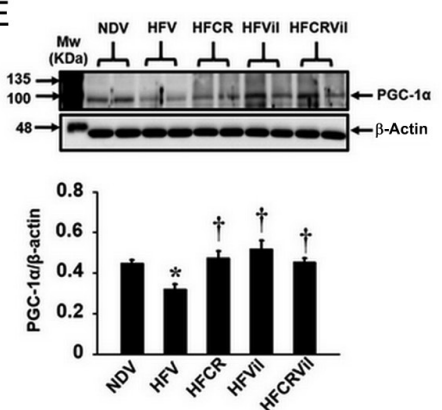

C

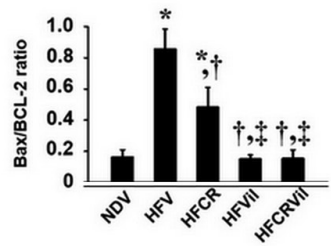

F
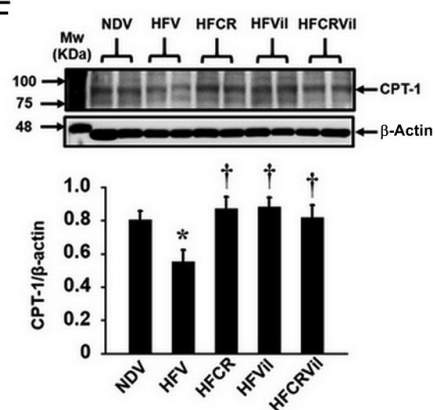

Figure 4

Effects of HFCR, vildagliptin and combined HFCR and vildagliptin on intracellular FGF21 signaling pathways including anti-apoptosis and fatty acid oxidation pathways. (A) Bax, (B) BCL-2, (C) Bax/BCL-2 ratio, (D) Cleaved Caspase-3/t-Caspase-3 ratio, (E) PGC-1 $\alpha$, and (F) CPT-1 in ND and HFD rats treated with vehicle, Caloric restriction (CR), vildagliptin, and CR combined vildagliptin. ${ }^{*} P<0.05$ vs NDV, ${ }^{\dagger} P<0.05$ vs HFV, ${ }^{\ddagger} P<0.05$ vs HFCR (1-WAY ANOVA, LSD post hoc test, $n=4-6$ per subgroups). CPT1, carnitine palmitoyltransferase 1; HFCR, high-fat diet treated with CR diet; HFCRVil, high-fat diet treated with CR diet and vildagliptin; HFD, high-fat diet; HFV, high-fat diet treated with vehicle; HFVil, high-fat diet treated with vildagliptin; ND, normal diet; NDV, normal diet treated with vehicle; PGC-1 $\alpha$, proliferator-activated receptor gamma coactivator 1-alpha.

ratio (Fig. 4C) and cleaved caspase-3 (Fig. 4D), when compared with the NDV group. Moreover, the HFV group also had significantly decreased anti-apoptotic signaling molecules as shown by decreased BCL-2 (Fig. 4B), when compared with the NDV group. Our results showed that HFCR rats had significantly decreased Bax (Fig. 4A), Bax/ BCL-2 ratio (Fig. 4C) and cleaved caspase-3 (Fig. 4D), when compared with the HFV group. Moreover, the HFCR group had significantly increased levels of BCL-2 (Fig. 4B), when compared with the HFV group. Interestingly, we found that vildagliptin-treated groups (HFVil and HFCRVil) had a greater reduction in Bax (Fig. 4A), Bax/BCL-2 ratio (Fig. 4C) and cleaved caspase-3 (Fig. 4D), when compared with the HFV and HFCR groups. Vildagliptin-treated groups (HFVil and HFCRVil) also had greater increased levels of BCL-2 (Fig. 4B), when compared with HFV and HFCR groups.

\section{CR and vildagliptin restored fatty acid $\beta$-oxidation (FAO) pathway protein expression in the heart}

At the end of 16 weeks of feeding on a HFD, the results in mitochondrial FAO pathways protein expression demonstrated that HFV rats had significantly decreased PGC-1 $\alpha$ (Fig. 4E) and CPT-1 (Fig. 4F) protein expression when compared with the NDV group. All treatments in HFCR, HFVil, and HFCRVil groups had led to significantly increased PGC-1 $\alpha$ (Fig. 4E) and CPT-1 (Fig. 4F) protein expression when compared with the HFV group.

\section{Vildagliptin exerted more effective cardiac mitochondrial protection than CR in obese-insulin- resistant rats}

At the end of 16 weeks of feeding on a HFD, the results regarding cardiac mitochondrial function demonstrated that HFD consumption significantly increased cardiac mitochondrial ROS production (Fig. 5A), decreased cardiac mitochondrial membrane potential changes verified by an attenuated red/green fluorescent intensity ratio $(\Delta \Psi$; Fig. 5B) and also increased cardiac mitochondrial swelling (Fig. 5C), when compared with the NDV group. HFCR, HFVil and HFCRVil groups had significantly decreased cardiac mitochondrial ROS production when compared with the HFV group (Fig. 5A). Moreover, the HFCR group had improved cardiac mitochondrial function identified by increased cardiac mitochondrial membrane potential changes $(\Delta \Psi$; Fig. $5 \mathrm{~B})$ and mitochondrial absorbance (Fig. 5C), when compared with the HFV groups.

Published by Bioscientifica Ltd 

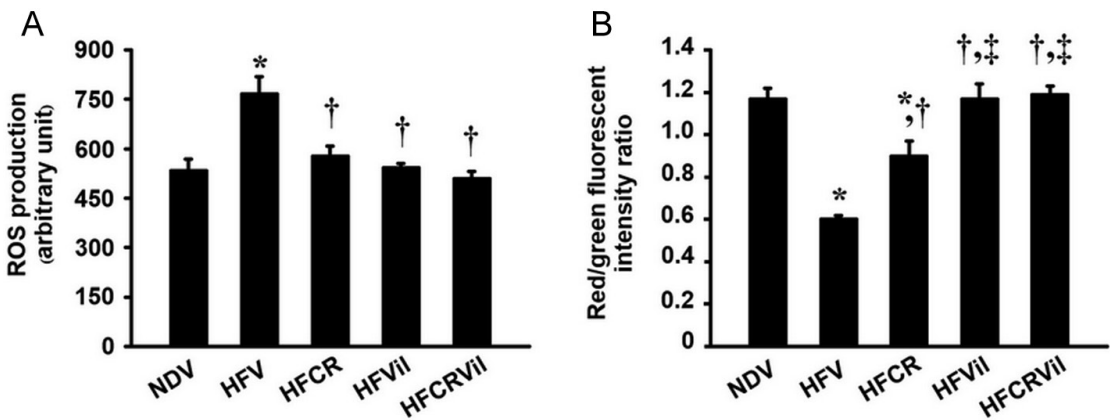
Figure 5
Effects of HFCR, vildagliptin and combined HFCR and vildagliptin on cardiac mitochondrial function and cardiac mitochondria morphology. (A) Cardiac mitochondrial ROS production, (B) red/green fluorescent intensity ratio, (C) Cardiac mitochondrial swelling, and (D) Cardiac mitochondria morphology by transmission electron microscope (TEM) in ND and HFD treated with vehicle, $C R$, vildagliptin, and $C R$ combined with vildagliptin. ${ }^{*} P<0.05$ vs NDV, ${ }^{+} P<0.05$ vs HFV, $\neq P<0.05$ vs HFCR (1-WAY ANOVA, LSD post hoc test, $n=6$ per subgroups). HFCR, high-fat diet treated with CR diet; HFCRVil, high-fat diet treated with CR diet and vildagliptin; HFD, high-fat diet; HFV, high-fat diet treated with vehicle; HFVil, high-fat diet treated with vildagliptin; ND, normal diet; NDV, normal diet treated with vehicle; ROS, reactive oxygen species.

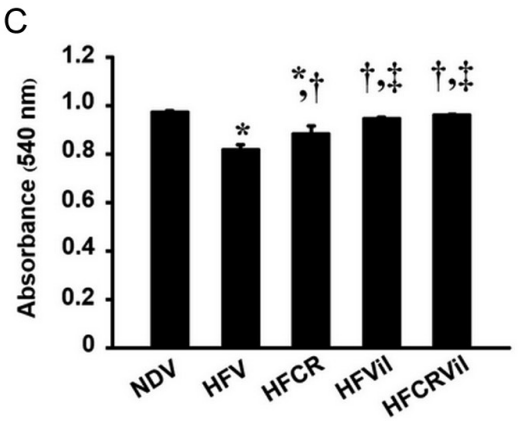

$\mathrm{D}$

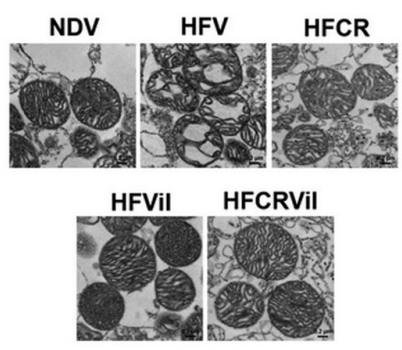

However, vildagliptin-treated groups (HFVil and HFCRVil) provided greater efficacy in improving cardiac mitochondrial function by restoring the cardiac mitochondrial membrane potential changes ( $\Delta \Psi$; Fig. $5 \mathrm{~B})$ and mitochondrial absorbance (Fig. 5C), when compared with the HFV and HFCR groups. Moreover, the cardiac mitochondrial morphology viewed by transmission electron microscope (TEM) demonstrated unfolded cristae in the mitochondria in the HFV group, indicating cardiac mitochondrial swelling in the HFV group. Although the cardiac mitochondrial morphology had improved in the HFCR group, when compared with the HFV group, this parameter had been restored to normal levels in vildagliptin-treated groups (HFVil and HFCRVil), when compared with HFV and HFCR groups (Fig. 5D).

\section{Discussion}

The major findings of this study are as follows: long-term HFD consumption led to obesity, insulin resistance, FGF21 resistance and increased oxidative stress levels. Moreover, high systolic blood pressure, cardiac mitochondrial dysfunction and cardiac sympathovagal imbalance were observed, and all of these factors contributed to LV dysfunction in obese-insulin-resistant rats. The three interventions in this study, including caloric restriction (CR), vildagliptin and combined CR and vildagliptin, had similar efficacy in improving insulin resistance, FGF21 resistance, metabolic disturbance, cardiac and serum MDA levels and mitochondrial ROS production. Interestingly, vildagliptin exerted greater cardioprotection than $\mathrm{CR}$ as vildagliptin appeared to reduce cardiac apoptosis, preserve cardiac mitochondrial membrane potential and attenuate cardiac mitochondrial swelling more effectively than CR. From all of these beneficial effects, vildagliptin effectively reduced blood pressure, improved cardiac sympathovagal imbalance and cardiac dysfunction in obese-insulinresistant rats.

In previous reports, 12-16 weeks of HFD consumption did not affect plasma triglyceride levels (Pratchayasakul et al. 2011, 2014). However, the excessive fat intake usually does lead to an accumulation of triglyceride (TG) in the liver and adipose tissues. This is due to the fact that it might take time to release (TG) from the liver and adipose tissue into the blood stream. Therefore, a longer duration of HFD feeding may be needed to increase plasma triglyceride levels as shown in previous reports (Manco et al. 2004, Gauthier et al. 2006, Hwang et al. 2010, Pratchayasakul et al. 2011, 2014).

In our obese-insulin-resistant rats, plasma glucose and triglyceride levels were not altered, compared to their baseline levels. This suggested that our obese-insulinresistant rats had hyperinsulinemia with euglycemia and normotriglyceridemia. Treatment with vildagliptin and caloric restriction did not affect plasma glucose and triglyceride levels. However, these interventions reduced plasma cholesterol and low-density lipoprotein (LDL) levels in obese-insulin-resistant rats. As the plasma levels 
of glucose and TG were not increased, both interventions did not interrupt the metabolism of glucose and TG at their physiological levels. Consistent with our previous reports in the obese-insulin-resistant model, our highfat fed rats were considered as pre-diabetic state rats (Pratchayasakul et al. 2011, Pongkan et al. 2016).

Regarding the effect of DDP4-inhibitor vildagliptin on FBG, reports from our group and others demonstrated that vildagliptin acted as a glycemic control agent without having a hypoglycemic effect (Yin et al. 2011, Maeda et al. 2012, Apaijai et al. 2014). This is due to the fact that vildagliptin reduced glucagon levels during hyperglycemia and at the same time did not inhibit any counter-regulatory glucagon response during hypoglycemia (Farngren et al. 2012). Moreover, vildagliptin also enhanced glucose sensitivity in alphacells by promoting the direct effect of low blood glucose levels to stimulate glucagon secretion (Ahren et al. 2011, Farngren et al. 2012). In addition, the increase of glucose-dependent insulinotropic polypeptide (GIP) was observed by vildagliptin therapy (Ahren et al. 2004, Mari et al. 2005, Farngren et al. 2012), thus stimulating glucagon secretion during low blood glucose levels (Meier et al. 2003, Christensen et al. 2011). Consistent with previous studies, our results showed that vildagliptin improved insulin sensitivity leading to reduced plasma insulin without affecting plasma glucose levels in obese-insulin-resistant rats (Apaijai et al. 2014, Pongkan et al. 2016).

Our previous studies have shown that long-term HFD consumption led to obese-insulin resistance with impaired cardiac sympathovagal balance and cardiac dysfunction (Apaijai et al. 2013, 2014). Previously, we proposed various mechanisms as being responsible for these impairments, such as metabolic disturbance, oxidative stress and cardiac mitochondrial dysfunction. In this study, we demonstrated that the HFD-fed rats had FGF21 resistance and impaired cardiac metabolism. FGF21 resistance was observed in our obese-insulinresistant rats and is associated with a reduction in FGF receptor function, as FGF receptor expression and $\beta$-Klotho expression were unaffected in our model. However, there are several discrepancies regarding the mechanism responsible for FGF21 resistance. Fisher and coworkers reported that mice fed with a high-fat/highsucrose diet for 22 weeks had FGF21 resistance and found that FGF21 receptor mRNA expression was reduced, whereas $\beta$-Klotho mRNA expression was unchanged in the liver of obese mice, compared to lean mice. However unlike in the liver, both FGF21 receptor and $\beta$-Klotho mRNA expression were reduced in white adipose tissue of obese mice, compared to lean mice (Fisher et al. 2010). In addition, our study on the HFD-induced obese-insulinresistant rat model demonstrated that HFD consumption did not alter FGF21 receptor protein expression in the heart (Tanajak et al. 2016). These findings suggest that the mechanism of FGF21 resistance is specific to the organ. However, Patel and coworkers reported that 12 weeks of HFD consumption reduced $\beta$-Klotho protein expression in the heart (Patel et al. 2014). Although they found that $\beta$-Klotho protein expression in the heart was decreased, the p-FGFR1 protein expression had not been investigated. Therefore, the role of HFD consumption of FGF21 receptors complex expression and function in the heart are still controversial. Future studies are needed to investigate the role of HFD consumption in FGF21 resistance, particularly with the changes of FGF21 receptor complex expression ( $\beta$-Klotho, FGFR1 and p-FGFR1) on the heart at different time points. In this model, however, the expression of $\beta$-klotho, the FGFR1 co-receptor, was unchanged. The decrease in FGF21 receptor complex function could lead to the impairment of the downstream signaling molecules, such as reduced ERK1/2 phosphorylation. Furthermore, we also found that the utilization of fatty acids was reduced in the HFD-fed rats as indicated by a reduction in PGC- $1 \alpha$ and CPT1 expression. Therefore, the FGF21 resistance and the alteration of cardiac metabolism could also contribute to cardiac dysfunction in our obese-insulin-resistant rats.

Besides ERK1/2, FGF21 receptor activation also regulates Akt, AMPK and adiponectin, which are known to determine cardiac metabolism. However, the definite mechanisms are still controversy. In cardiac ischemiareperfusion injury model, it has been shown that FGF21 administration exerted cardioprotection via the activation of Akt and AMPK (Patel et al. 2014). However, $\mathrm{Xu}$ and coworkers demonstrated that FGF21 therapy did not alter Akt in the insulin-resistant C57BL6 mice heart (Xu et al. 2009). However, our results showed that there was no difference in the p-Akt/t-Akt ratio between all groups. This could be due to the use of different models and drugs in these studies. Regarding adiponectin, Joki and coworkers demonstrated that activation of FGF21 receptors increased plasma adiponectin, which contributed to reduced cardiac inflammation and exerted cardioprotection against cardiac remodeling in the myocardial infarction mice model (Joki et al. 2015). http://joe.endocrinology-journals.org DOI: $10.1530 / J O E-16-0406$ (c) 2017 Society for Endocrinology Printed in Great Britain
Published by Bioscientifica Ltd 
Future studies are needed to investigate the roles of FGF21 receptor activation on AMPK and adiponectin in obeseinsulin-resistant condition.

In this study, although all interventions effectively reduced insulin resistance, only CR led to reduced body weight gain in obese-insulin-resistant rats. This is due to a reduction of caloric intake, which led to reduced visceral fat deposition. Our findings are consistent with previous studies, which also showed that CR reduced body weight gain in various animal models (Wilsey \& Scarpace 2004, Park et al. 2006, Niemann et al. 2010, Takatsu et al. 2013), whereas vildagliptin improved insulin resistance without any changes in body weight (Pipatpiboon et al. 2013, Apaijai et al. 2014). In addition, there is growing evidence that cardiac mitochondrial dysfunction impairs cardiac function and cardiac autonomic function. It has been reported that there is a relationship between cardiac mitochondrial dysfunction, cardiac dysfunction and cardiac autonomic imbalance in diabetic patients (Momiyama et al. 2002). In this study, vildagliptin led to greater improvement in cardiac mitochondrial function than CR in obese-insulin-resistant rats. Although both vildagliptin and $\mathrm{CR}$ have similar effects on oxidative stress, including on levels of MDA and mitochondrial ROS, vildagliptin attenuated cardiac mitochondrial membrane depolarization and mitochondrial swelling more effectively than CR. These findings indicate that vildagliptin exerted greater cardioprotection by protecting mitochondrial dysfunction more effectively than CR in obese-insulin-resistant rats.

Moreover, it has been shown that mitochondria could regulate the apoptotic process. In this study, we found that vildagliptin appeared to reduce cardiac apoptosis more effectively than CR in the obese-insulinresistant model as shown by greater reduction of Bax and cleaved caspase-3 expression and higher BCL-2 expression. This in turn would have then caused reduced Bax translocation to the mitochondrial outer membrane. Previous reports have shown that Bax translocation after stress stimuli caused mitochondrial membrane depolarization (Smaili et al. 2001). Furthermore, Bax binds with voltage-dependent anion channels (VDAC), which is followed by conformational change of the mitochondrial permeability transition pores (mPTP). This process allows proton and water influx across the mitochondrial membrane into the mitochondrial matrix, leading to mitochondrial swelling. These mechanisms could be responsible for the superior effects of vildagliptin to $\mathrm{CR}$ on cardiac function and cardiac autonomic function in obese-insulin-resistant rats.
In this study, we also demonstrated that chronic HFD consumption is related to FGF21 resistance. FGF21 has been shown to regulate cardiac function in various models (Cong et al. 2013, Planavila et al. 2013, 2014, Tanajak et al. 2015, Yan et al. 2015). Our results showed that both CR and vildagliptin improved FGF21 resistance by improving FGF21 receptor function and its downstream signaling. These beneficial effects could also contribute to improved LV function in obese-insulin-resistant rats observed in this study. Although the vildagliptin-treated group demonstrated greater efficacy than caloric restriction in improving anti-apoptotic pathways and attenuating cardiac mitochondrial dysfunction, the combination of vildagliptin and caloric restriction did not provide an additional effect. It is possible that vildagliptin therapy already had a superior effect to caloric restriction as our results showed that vildagliptin could reverse the metabolic and cardiac adverse effects caused by longterm HFD consumption back to the normal physiological levels, whereas this restoration was not observed in the caloric restriction group.

In conclusion, chronic HFD consumption leads to obese-insulin resistance and FGF21 resistance. Although CR is effective in improving metabolic regulation and FGF21 sensitivity, vildagliptin provides greater efficacy in preventing cardiac dysfunction by improving antiapoptotic pathways and attenuating cardiac mitochondrial dysfunction in obese-insulin-resistant rats.

\section{Declaration of interest}

The authors declare that there is no conflict of interest that could be perceived as prejudicing the impartiality of the research reported.

\section{Funding}

This work was supported by a NSTDA Research Chair Grant from the National Science and Technology Development Agency Thailand (NC), the Thailand Research Fund Royal Golden Jubilee Program (PHD/0078/2557 PT\&SC), BRG5780016 (SC) and Chiang Mai University Center of Excellence Award (NC).

\section{Author contribution statement}

$\mathrm{S} \mathrm{C}$ and $\mathrm{N} C$ conceived and designed the experiments. $\mathrm{P} T$ and $\mathrm{H} P$ conducted the experiments. P T, H P, N S, J K, N A, S C and N C analyzed the data. P T, N A, S C and N C wrote the manuscript.

\section{Acknowledgements}

The authors would like to thank Piangkwan Sa-nguanmoo for her technical assistance on metabolic parameter assessment and Cicely Proctor for her editorial assistance of this paper.

Published by Bioscientifica Ltd. 


\section{References}

Ahren B, Landin-Olsson M, Jansson PA, Svensson M, Holmes D \& Schweizer A 2004 Inhibition of dipeptidyl peptidase-4 reduces glycemia, sustains insulin levels, and reduces glucagon levels in type 2 diabetes. Journal of Clinical Endocrinology and Metabolism 89 2078-2084. (doi:10.1210/jc.2003-031907)

Ahren B, Schweizer A, Dejager S, Villhauer EB, Dunning BE \& Foley JE 2011 Mechanisms of action of the dipeptidyl peptidase- 4 inhibitor vildagliptin in humans. Diabetes, Obesity and Metabolism 13 775-783. (doi:10.1111/j.1463-1326.2011.01414.x)

Apaijai N, Pintana H, Chattipakorn SC \& Chattipakorn N 2012 Cardioprotective effects of metformin and vildagliptin in adult rats with insulin resistance induced by a high-fat diet. Endocrinology 153 3878-3885. (doi:10.1210/en.2012-1262)

Apaijai N, Pintana H, Chattipakorn SC \& Chattipakorn N 2013 Effects of vildagliptin versus sitagliptin, on cardiac function, heart rate variability and mitochondrial function in obese insulin-resistant rats. British Journal of Pharmacology 169 1048-1057. (doi:10.1111/bph.12176)

Apaijai N, Chinda K, Palee S, Chattipakorn S \& Chattipakorn N 2014 Combined vildagliptin and metformin exert better cardioprotection than monotherapy against ischemia-reperfusion injury in obeseinsulin resistant rats. PLOS ONE 9 e102374. (doi:10.1371/journal. pone.0102374)

Aydin C \& Gordon CJ 2013 Thermoregulatory, cardiovascular, and metabolic responses to mild caloric restriction in the Brown Norway rat. Physiological Reports 1 e00016. (doi:10.1002/phy2.16)

Burkey BF, Li X, Bolognese L, Balkan B, Mone M, Russell M, Hughes TE \& Wang PR 2005 Acute and chronic effects of the incretin enhancer vildagliptin in insulin-resistant rats. Journal of Pharmacology and Experimental Therapeutics 315 688-695. (doi:10.1124/jpet.105.087064)

Chattipakorn N, Incharoen T, Kanlop N \& Chattipakorn S 2007 Heart rate variability in myocardial infarction and heart failure. International Journal of Cardiology 120 289-296. (doi:10.1016/j.ijcard.2006.11.221)

Chau MD, Gao J, Yang Q, Wu Z \& Gromada J 2010 Fibroblast growth factor 21 regulates energy metabolism by activating the AMPKSIRT1-PGC-1alpha pathway. PNAS 107 12553-12558. (doi:10.1073/ pnas.1006962107)

Chinda K, Palee S, Surinkaew S, Phornphutkul M, Chattipakorn S \& Chattipakorn N 2013 Cardioprotective effect of dipeptidyl peptidase-4 inhibitor during ischemia-reperfusion injury. International Journal of Cardiology 167 451-457. (doi:10.1016/j.ijcard.2012.01.011)

Chinda K, Sanit J, Chattipakorn S \& Chattipakorn N 2014 Dipeptidyl peptidase- 4 inhibitor reduces infarct size and preserves cardiac function via mitochondrial protection in ischaemia-reperfusion rat heart. Diabetes and Vascular Disease Research 11 75-83. (doi:10.1177/1479164113516134)

Christensen M, Vedtofte L, Holst JJ, Vilsboll T \& Knop FK 2011 Glucosedependent insulinotropic polypeptide: a bifunctional glucosedependent regulator of glucagon and insulin secretion in humans. Diabetes 60 3103-3109. (doi:10.2337/db11-0979)

Cong WT, Ling J, Tian HS, Ling R, Wang Y, Huang BB, Zhao T, Duan YM, Jin LT \& Li XK 2013 Proteomic study on the protective mechanism of fibroblast growth factor 21 to ischemia-reperfusion injury. Canadian Journal of Physiology and Pharmacology 91 973-984. (doi:10.1139/cjpp2012-0441)

Dolinsky VW, Morton JS, Oka T, Robillard-Frayne I, Bagdan M, Lopaschuk GD, Des Rosiers C, Walsh K, Davidge ST \& Dyck JR 2010 Calorie restriction prevents hypertension and cardiac hypertrophy in the spontaneously hypertensive rat. Hypertension 56 412-421. (doi:10.1161/HYPERTENSIONAHA.110.154732)

Farngren J, Persson M, Schweizer A, Foley JE \& Ahren B 2012 Vildagliptin reduces glucagon during hyperglycemia and sustains glucagon counterregulation during hypoglycemia in type 1 diabetes. Journal of Clinical Endocrinology and Metabolism 97 3799-3806. (doi:10.1210/ jc.2012-2332)
Feng M, Whitesall S, Zhang Y, Beibel M, D'Alecy L \& DiPetrillo K 2008 Validation of volume-pressure recording tail-cuff blood pressure measurements. American Journal of Hypertension 21 1288-1291. (doi:10.1038/ajh.2008.301)

Fisher FM, Chui PC, Antonellis PJ, Bina HA, Kharitonenkov A, Flier JS \& Maratos-Flier E 2010 Obesity is a fibroblast growth factor 21 (FGF21)resistant state. Diabetes 59 2781-2789. (doi:10.2337/db10-0193)

Fontana L, Meyer TE, Klein S \& Holloszy JO 2004 Long-term calorie restriction is highly effective in reducing the risk for atherosclerosis in humans. PNAS 101 6659-6663. (doi:10.1073/pnas.0308291101)

Friedewald WT, Levy RI \& Fredrickson DS 1972 Estimation of the concentration of low-density lipoprotein cholesterol in plasma, without use of the preparative ultracentrifuge. Clinical Chemistry $\mathbf{1 8}$ 499-502.

Gauthier MS, Favier R \& Lavoie JM 2006 Time course of the development of non-alcoholic hepatic steatosis in response to high-fat diet-induced obesity in rats. British Journal of Nutrition 95 273-281. (doi:10.1079/ BJN20051635)

Hwang LL, Wang CH, Li TL, Chang SD, Lin LC, Chen CP, Chen CT, Liang KC, Ho IK, Yang WS, et al. 2010 Sex differences in highfat diet-induced obesity, metabolic alterations and learning, and synaptic plasticity deficits in mice. Obesity 18 463-469. (doi:10.1038/ oby.2009.273)

Inthachai T, Lekawanvijit S, Kumfu S, Apaijai N, Pongkan W, Chattipakorn SC \& Chattipakorn N 2015 Dipeptidyl peptidase-4 inhibitor improves cardiac function by attenuating adverse cardiac remodelling in rats with chronic myocardial infarction. Experimental Physiology 100 667-679. (doi:10.1113/EP085108)

Inzucchi SE, Bergenstal RM, Buse JB, Diamant M, Ferrannini E, Nauck M Peters AL, Tsapas A, Wender R \& Matthews DR 2015 Management of hyperglycemia in type 2 diabetes, 2015: a patient-centered approach: update to a position statement of the American Diabetes Association and the European Association for the Study of Diabetes. Diabetes Care 38 140-149. (doi:10.2337/dc14-2441)

Itoh N \& Ornitz DM 2011 Fibroblast growth factors: from molecular evolution to roles in development, metabolism and disease. Journal of Biochemistry 149 121-130. (doi:10.1093/jb/mvq121)

Joki Y, Ohashi K, Yuasa D, Shibata R, Ito M, Matsuo K, Kambara T, Uemura Y, Hayakawa S, Hiramatsu-Ito M, et al. 2015 FGF21 attenuates pathological myocardial remodeling following myocardial infarction through the adiponectin-dependent mechanism. Biochemical and Biophysical Research Communications 459 124-130. (doi:10.1016/j. bbrc.2015.02.081)

Kharitonenkov A 2009 FGFs and metabolism. Current Opinion in Pharmacology 9 805-810. (doi:10.1016/j.coph.2009.07.001)

Kilkenny C, Browne W, Cuthill IC, Emerson M, Altman DG \& NC3Rs Reporting Guidelines Working Group 2010 Animal research: reporting in vivo experiments: the ARRIVE guidelines. British Journal of Pharmacology 160 1577-1579. (doi:10.1111/j.14765381.2010.00872.x)

Liu SQ, Tefft BJ, Roberts DT, Zhang LQ, Ren Y, Li YC, Huang Y, Zhang D, Phillips HR \& Wu YH 2012 Cardioprotective proteins upregulated in the liver in response to experimental myocardial ischemia. American Journal of Physiology: Heart and Circulatory Physiology $\mathbf{3 0 3}$ H1446-H1458. (doi:10.1152/ajpheart.00362.2012)

Liu SQ, Roberts D, Kharitonenkov A, Zhang B, Hanson SM, Li YC, Zhang LQ \& Wu YH 2013 Endocrine protection of ischemic myocardium by FGF21 from the liver and adipose tissue. Scientific Reports 3 2767. (doi:10.1038/srep02767)

Lu Y, Liu JH, Zhang LK, Du J, Zeng XJ, Hao G, Huang J, Zhao DH, Wang GZ \& Zhang YC 2010 Fibroblast growth factor 21 as a possible endogenous factor inhibits apoptosis in cardiac endothelial cells Chinese Medical Journal 123 3417-3421. (doi:10.3760/cma.j.issn. 0366-6999.2010.23.008)

Maeda S, Matsui T \& Yamagishi S 2012 Vildagliptin inhibits oxidative stress and vascular damage in streptozotocin-induced diabetic rats. http://joe.endocrinology-journals.org

DOI: $10.1530 / \mathrm{JOE}-16-0406$
() 2017 Society for Endocrinology Printed in Great Britain 
International Journal of Cardiology 158 171-173. (doi:10.1016/j. ijcard.2012.04.087)

Manco M, Calvani M \& Mingrone G 2004 Effects of dietary fatty acids on insulin sensitivity and secretion. Diabetes, Obesity and Metabolism 6 402-413. (doi:10.1111/j.1462-8902.2004.00356.x)

Mari A, Sallas WM, He YL, Watson C, Ligueros-Saylan M, Dunning BE, Deacon CF, Holst JJ \& Foley JE 2005 Vildagliptin, a dipeptidyl peptidase-IV inhibitor, improves model-assessed beta-cell function in patients with type 2 diabetes. Journal of Clinical Endocrinology and Metabolism 90 4888-4894. (doi:10.1210/jc.2004-2460)

Meier JJ, Gallwitz B, Siepmann N, Holst JJ, Deacon CF, Schmidt WE \& Nauck MA 2003 Gastric inhibitory polypeptide (GIP) dosedependently stimulates glucagon secretion in healthy human subjects at euglycaemia. Diabetologia 46 798-801. (doi:10.1007/s00125-0031103-y)

Momiyama Y, Suzuki Y, Ohtomo M, Atsumi Y, Matsuoka K, Ohsuzu F \& Kimura M 2002 Cardiac autonomic nervous dysfunction in diabetic patients with a mitochondrial DNA mutation: assessment by heart rate variability. Diabetes Care 25 2308-2313. (doi:10.2337/ diacare.25.12.2308)

Niemann B, Chen Y, Issa H, Silber RE \& Rohrbach S 2010 Caloric restriction delays cardiac ageing in rats: role of mitochondria. Cardiovascular Research 88 267-276. (doi:10.1093/cvr/cvq273)

Ong KL, Januszewski AS, O'Connell R, Jenkins AJ, Xu A, Sullivan DR, Barter PJ, Hung WT, Scott RS, Taskinen MR, et al. 2015 The relationship of fibroblast growth factor 21 with cardiovascular outcome events in the Fenofibrate Intervention and Event Lowering in Diabetes study. Diabetologia 58 464-473. (doi:10.1007/s00125-0143458-7)

Park S, Komatsu T, Hayashi H, Yamaza H, Chiba T, Higami Y, Kuramoto K \& Shimokawa I 2006 Calorie restriction initiated at middle age improved glucose tolerance without affecting age-related impairments of insulin signaling in rat skeletal muscle. Experimental Gerontology 41 837-845. (doi:10.1016/j.exger.2006.06.055)

Patel V, Adya R, Chen J, Ramanjaneya M, Bari MF, Bhudia SK, Hillhouse EW, Tan BK \& Randeva HS 2014 Novel insights into the cardio-protective effects of FGF21 in lean and obese rat hearts. PLoS ONE 9 e87102. (doi:10.1371/journal.pone.0087102)

Pipatpiboon N, Pratchayasakul W, Chattipakorn N \& Chattipakorn SC 2012 PPARgamma agonist improves neuronal insulin receptor function in hippocampus and brain mitochondria function in rats with insulin resistance induced by long term high-fat diets. Endocrinology 153 329-338. (doi:10.1210/en.2011-1502)

Pipatpiboon N, Pintana H, Pratchayasakul W, Chattipakorn N \& Chattipakorn SC 2013 DPP4-inhibitor improves neuronal insulin receptor function, brain mitochondrial function and cognitive function in rats with insulin resistance induced by high-fat diet consumption. European Journal of Neuroscience 37 839-849. (doi:10.1111/ejn.12088)

Planavila A, Redondo I, Hondares E, Vinciguerra M, Munts C, Iglesias R, Gabrielli LA, Sitges M, Giralt M, van Bilsen M, et al. 2013 Fibroblast growth factor 21 protects against cardiac hypertrophy in mice. Nature Communications 4 2019. (doi:10.1038/ncomms3019)

Planavila A, Redondo-Angulo I, Ribas F, Garrabou G, Casademont J, Giralt M \& Villarroya F 2014 Fibroblast growth factor 21 protects the heart from oxidative stress. Cardiovascular Research 106 19-31. (doi:10.1093/cvr/cvu263)

Pongchaidecha A, Lailerd N, Boonprasert W \& Chattipakorn N 2009 Effects of curcuminoid supplement on cardiac autonomic status in high-fat-induced obese rats. Nutrition 25 870-878. (doi:10.1016/j. nut.2009.02.001)

Pongkan W, Pintana H, Jaiwongkam T, Kredphoo S, Sivasinprasasn S, Chattipakorn SC \& Chattipakorn N 2016 Vildagliptin reduces cardiac ischemic-reperfusion injury in obese orchiectomized rats. Journal of Endocrinology 231 81-95. (doi:10.1530/JOE-16-0232)
Pratchayasakul W, Kerdphoo S, Petsophonsakul P, Pongchaidecha A, Chattipakorn N \& Chattipakorn SC 2011 Effects of high-fat diet on insulin receptor function in rat hippocampus and the level of neuronal corticosterone. Life Science 88 619-627. (doi:10.1016/j. lfs.2011.02.003)

Pratchayasakul W, Chattipakorn N \& Chattipakorn SC 2014 Estrogen restores brain insulin sensitivity in ovariectomized non-obese rats, but not in ovariectomized obese rats. Metabolism 63 851-859. (doi:10.1016/j.metabol.2014.03.009)

Shen Y, Ma X, Zhou J, Pan X, Hao Y, Zhou M, Lu Z, Gao M, Bao Y \& Jia W 2013 Additive relationship between serum fibroblast growth factor 21 level and coronary artery disease. Cardiovascular Diabetology 12124. (doi:10.1186/1475-2840-12-124)

Smaili SS, Hsu YT, Sanders KM, Russell JT \& Youle RJ 2001 Bax translocation to mitochondria subsequent to a rapid loss of mitochondrial membrane potential. Cell Death and Differentiation $\mathbf{8}$ 909-920. (doi:10.1038/sj.cdd.4400889)

Surinkaew S, Kumphune S, Chattipakorn S \& Chattipakorn N 2013 Inhibition of p38 MAPK during ischemia, but not reperfusion, effectively attenuates fatal arrhythmia in ischemia/reperfusion heart. Journal of Cardiovascular Pharmacology 61 133-141. (doi:10.1097/ FJC.0b013e318279b7b1)

Takatsu M, Nakashima C, Takahashi K, Murase T, Hattori T, Ito H, Murohara T \& Nagata K 2013 Calorie restriction attenuates cardiac remodeling and diastolic dysfunction in a rat model of metabolic syndrome. Hypertension 62 957-965. (doi:10.1161/ HYPERTENSIONAHA.113.02093)

Tan BK, Hallschmid M, Adya R, Kern W, Lehnert H \& Randeva HS 2011 Fibroblast growth factor 21 (FGF21) in human cerebrospinal fluid: relationship with plasma FGF21 and body adiposity. Diabetes 60 2758-2762. (doi:10.2337/db11-0672)

Tanajak P, Chattipakorn SC \& Chattipakorn N 2015 Effects of fibroblast growth factor 21 on the heart. Journal of Endocrinology 227 R13-R30. (doi:10.1530/JOE-15-0289)

Tanajak P, Sa-Nguanmoo P, Wang X, Liang G, Li X, Jiang C, Chattipakorn SC \& Chattipakorn N 2016 Fibroblast growth factor 21 (FGF21) therapy attenuates left ventricular dysfunction and metabolic disturbance by improving FGF21 sensitivity, cardiac mitochondrial redox homoeostasis and structural changes in pre-diabetic rats. Acta Physiologica 217 287-299. (doi:10.1111/apha.12698)

Thummasorn S, Kumfu S, Chattipakorn S \& Chattipakorn N 2011 Granulocyte-colony stimulating factor attenuates mitochondrial dysfunction induced by oxidative stress in cardiac mitochondria. Mitochondrion 11 457-466. (doi:10.1016/j.mito.2011.01.008)

Wente W, Efanov AM, Brenner M, Kharitonenkov A, Koster A, Sandusky GE, Sewing S, Treinies I, Zitzer H \& Gromada J 2006 Fibroblast growth factor-21 improves pancreatic beta-cell function and survival by activation of extracellular signal-regulated kinase 1/2 and Akt signaling pathways. Diabetes 55 2470-2478. (doi:10.2337/ db05-1435)

Wilsey J \& Scarpace PJ 2004 Caloric restriction reverses the deficits in leptin receptor protein and leptin signaling capacity associated with diet-induced obesity: role of leptin in the regulation of hypothalamic long-form leptin receptor expression. Journal of Endocrinology $\mathbf{1 8 1}$ 297-306. (doi:10.1677/joe.0.1810297)

Wu X, Qi YF, Chang JR, Lu WW, Zhang JS, Wang SP, Cheng SJ, Zhang M, Fan Q, Lv Y, et al. 2014 Possible role of fibroblast growth factor 21 on atherosclerosis via amelioration of endoplasmic reticulum stressmediated apoptosis in apoE mice. Heart and Vessels 30 657-668. (doi:10.1007/s00380-014-0557-9)

Wycherley TP, Brinkworth GD, Noakes M, Buckley JD \& Clifton PM 2008 Effect of caloric restriction with and without exercise training on oxidative stress and endothelial function in obese subjects with type 2 diabetes. Diabetes, Obesity and Metabolism $101062-1073$. (doi:10.1111/j.1463-1326.2008.00863.x) http://joe.endocrinology-journals.org

DOI: $10.1530 / J O E-16-0406$
() 2017 Society for Endocrinology Printed in Great Britain 
Xu J, Stanislaus S, Chinookoswong N, Lau YY, Hager T, Patel J, Ge H, Weiszmann J, Lu SC, Graham M, et al. 2009 Acute glucose-lowering and insulin-sensitizing action of FGF21 in insulin-resistant mouse models - association with liver and adipose tissue effects. American Journal of Physiology: Endocrinology and Metabolism 297 E1105-E1114. (doi:10.1152/ajpendo.00348.2009)

Yan X, Chen J, Zhang C, Zhou S, Zhang Z, Chen J, Feng W, Li X \& Tan Y 2015 FGF21 deletion exacerbates diabetic cardiomyopathy by aggravating cardiac lipid accumulation. Journal of Cellular and Molecular Medicine 19 1557-1568. (doi:10.1111/jcmm.12530)

Yin M, Sillje HH, Meissner M, van Gilst WH \& de Boer RA 2011 Early and late effects of the DPP-4 inhibitor vildagliptin in a rat model of post- myocardial infarction heart failure. Cardiovascular Diabetology 1085. (doi:10.1186/1475-2840-10-85)

Yu Y, Bai F, Liu Y, Yang Y, Yuan Q, Zou D, Qu S, Tian G, Song L, Zhang T, et al. 2015 Fibroblast growth factor (FGF21) protects mouse liver against D-galactose-induced oxidative stress and apoptosis via activating Nrf2 and PI3K/Akt pathways. Molecular and Cellular Biochemistry 403 287-299. (doi:10.1007/s11010-015-2358-6)

Zhang C, Huang Z, Gu J, Yan X, Lu X, Zhou S, Wang S, Shao M, Zhang F, Cheng P, et al. 2015 Fibroblast growth factor 21 protects the heart from apoptosis in a diabetic mouse model via extracellular signalregulated kinase 1/2-dependent signalling pathway. Diabetologia 58 1937-1948. (doi:10.1007/s00125-015-3630-8)

Received in final form 19 October 2016

Accepted 13 November 2016

Accepted Preprint published online 14 November 2016
Published by Bioscientifica Ltd. 